\title{
Malignant Phyllodes Tumour with Liposarcomatous Differentiation, Invasive Tubular Carcinoma, and Ductal and Lobular Carcinoma In Situ: Case Report and Review of the Literature
}

\author{
Mardiana Abdul Aziz, ${ }^{1}$ Frank Sullivan, ${ }^{2}$ Michael J. Kerin, ${ }^{3}$ and Grace Callagy ${ }^{1,4}$ \\ ${ }^{1}$ Division of Anatomic Pathology, Galway University Hospitals, Galway, Ireland \\ ${ }^{2}$ Department of Radiation Oncology, Galway University Hospitals, Galway, Ireland \\ ${ }^{3}$ Department of Surgery, Galway University Hospitals, Galway, Ireland \\ ${ }^{4}$ Division of Pathology, Clinical Science Institute, National University of Ireland, Galway, Costello Road, Galway, Ireland
}

Correspondence should be addressed to Grace Callagy, grace.callagy@nuigalway.ie

Received 20 December 2009; Accepted 11 April 2010

Academic Editor: Gary M. K. Tse

Copyright ( $\odot 2010$ Mardiana Abdul Aziz et al. This is an open access article distributed under the Creative Commons Attribution License, which permits unrestricted use, distribution, and reproduction in any medium, provided the original work is properly cited.

A 43-year-old woman presented with a right breast lump that had enlarged over 5 months. She had chemoradiotherapy for nonHodgkin's lymphoma in 1989. Histology revealed a malignant phyllodes tumour (PT) with liposarcomatous differentiation and ductal carcinoma in situ (DCIS) within the tumour with invasive tubular carcinoma, DCIS, and lobular carcinoma in situ in the surrounding breast. She had surgery and adjuvant radiotherapy. One year follow-up showed no recurrence or metastatic disease. Liposarcomatous differentiation is uncommon in PTs, and coexisting carcinoma is rare with 38 cases in 31 reports in the literature. Carcinoma is reported in malignant $(n=19)$, benign $(n=16)$ and in borderline PTs $(n=3)$ with invasive carcinoma $(n=18)$ and pure in situ carcinoma $(n=19)$ recorded in equal frequency. Carcinoma is more commonly found within the confines of benign PTs; whereas it is more often found surrounding the PT or in the contralateral breast in malignant PTs. Previous radiotherapy treatment is reported in only two cases. The aetiology of co-existing carcinoma is unclear but the rarity of previous radiotherapy treatment suggests that it is incidental. This case highlights the diverse pathology that can occur with PTs, which should be considered when evaluating pathology specimens as they may impact on patient management.

\section{Introduction}

Phyllodes tumors (PTs) of the breast are uncommon biphasic fibroepithelial neoplasms that account for $<1 \%$ of all breast tumours. Most PTs are benign and carry a risk of local recurrence whereas malignant PTs have a $13 \%$ risk of haematogenous metastasis [1]. The distinction between benign, borderline and malignant PT is based on the assessment of a number of histological features including infiltrative margin, stromal overgrowth, stromal atypia, cellularity, and mitotic activity. However, while histological features are helpful, they are not accurate predictors of tumour behavior, and no single parameter is reliable in all cases [2].
PTs are believed to arise from intralobular or periductal stroma and may arise de novo or from pre-existing fibroadenomas [2]. Up to $30 \%$ of PTs show malignant transformation, most often in the form of malignant transformation of the stroma, which usually shows fibrosarcomatous differentiation and rarely heterologous sarcomatous elements. Malignant transformation of epithelial elements is very rare with only 38 cases reported in the literature. We present a case of a malignant PT that contained heterologous liposarcomatous stromal differentiation and exhibited a range of epithelial pathology. Ductal carcinoma in situ (DCIS) was present within the PT; DCIS, invasive tubular carcinoma, and multiple foci of lobular carcinoma in situ (LCIS) were noted in the adjacent breast tissue. Although it is generally accepted 


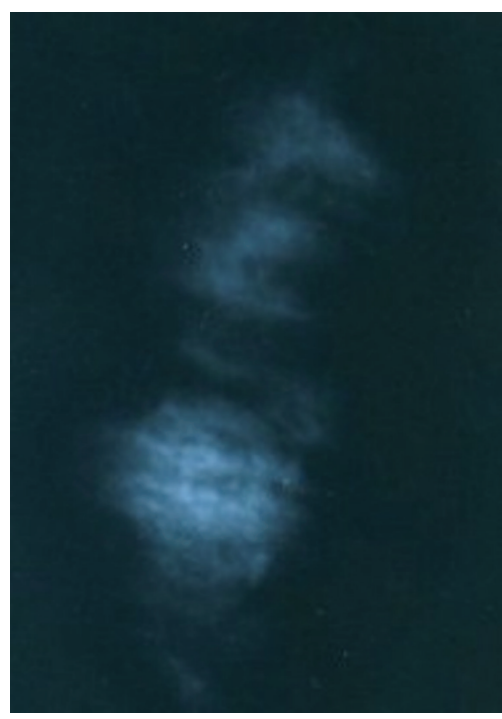

Figure 1: Mammogram of the left breast. A relatively wellcircumscribed mass was present in lower central aspect of the breast.

that the prognosis of a patient with a malignant PT depends on the nature of the PT, malignant epithelial components should, if present, be taken into account when managing the patient.

\section{Case Report}

A 43-year-old woman presented with a palpable lump in the central aspect of the left breast below the nipple. The lump was present for five months. It had increased in size over that time and was tender. Mammography showed a relatively well-circumscribed $3.4 \mathrm{~cm}$ mass in the lower central aspect of the breast (Figure 1). An ultrasound-guided biopsy was performed and showed a fibroepithelial lesion with increased stromal cellularity and nuclear atypia, consistent with a PT. The patient had a history of non-Hodgkin's lymphoma involving the lumbar spine in 1989, which was treated with chemotherapy and radiotherapy to the lumbar area.

A wide local excision of the left breast was performed which was followed by a wider cavity excision and latissimus dorsi flap reconstruction. The patient was given adjuvant radiotherapy. The breast received $50.4 \mathrm{~Gy}$ at $1.8 \mathrm{~Gy}$ per fraction with a cone-down boost to the tumour bed, giving a total dose of $63 \mathrm{~Gy}$ to the area considered at the greatest risk of recurrence. Follow-up after one year showed no evidence of recurrence or metastasis. Axillary sampling was not performed but no axillary lymphadenopathy was identified at one year follow-up.

\section{Pathological Findings}

The wide local excision of the left breast, $75 \times 60 \times 30 \mathrm{~mm}$, contained a well-defined, partly encapsulated tumor, $25 \times 35 \times 27 \mathrm{~mm}$, with a papillary texture. A PT was confirmed histologically. The tumour showed the characteristic enhanced intracanalicular growth pattern with leaf-like projections into dilated cysts, extensive stromal overgrowth, and marked stromal hypercellularity (Figure 2(a)). Frankly malignant stromal features including nuclear pleomorphism and a high mitotic count (19 per 10 high power fields) were seen (Figure 2(b)). In many areas, liposarcomatous differentiation, characterized by pleomorphic lipoblasts, was present (Figure 2(c)). The margins of the PT were focally infiltrative. The epithelial component within the PT exhibited hyperplasia of usual type as well as foci of DCIS, intermediate grade, with cribriform and solid growth patterns, without necrosis (Figure 2(d)). A small invasive carcinoma, $2 \mathrm{~mm}$ in size, was present at the periphery of the PT close to the deep margin of the specimen (Figure 3(a)). This was composed of well-formed tubules that lacked a myoepithelial layer (Smooth Muscle Heavy Chain Myosin and p63 negative) and was regarded as a grade 1 tumour [3] although it was too small to grade accurately based on the absence of sufficient high power fields for scoring of mitotic activity. Oestrogen receptor was strongly expressed and Her-2 was negative. The invasive tumour was adjacent to a small duct with morphological features of low-grade DCIS but there was no morphological transition between invasive carcinoma and the stromal or epithelial components of the adjacent PT. Multiple scattered foci of LCIS were also present peripheral to the PT, confirmed by downregulation of E-Cadherin staining (Figures 3(b) and 3(c)). The scattered nature of the LCIS precludes accurate measurement of its size.

The cavity re-excision specimen $(190 \mathrm{~g}, 98 \times 97 \times$ $47 \mathrm{~mm}$ ) included skeletal muscle and showed no residual PT. A single focus of DCIS, high-nuclear grade with comedonecrosis and microcalcification, $3 \mathrm{~mm}$ in size, was present within $1 \mathrm{~mm}$ to the medial margin (Figure 3(d)). Multiple foci of LCIS were also noted.

\section{Discussion and Review}

Metaplastic change within PTs is uncommon. In the largest series of PTs reported, stromal metaplasia was present in only 11 of 335 cases, and malignant transformation of epithelium in the form of DCIS and LCIS was seen in only two cases [17]. Stromal changes included adipose and chondromyxoid elements and were seen in benign and borderline PTs whereas malignant heterologous elements were reported in malignant PTs. The latter was most commonly in the form of liposarcomatous differentiation $[17,20,24,30,33$, 34 ], which does not equate with more aggressive clinical behavior [30, 35]. Other forms of heterologous change are reported in PTs including osteosarcoma, rhabdomyosarcomas, leiomyosarcoma, rhabdomyosarcoma, and angiosarcoma $[17,36]$. Epithelial change in the form of usual-type epithelial hyperplasia is well recognized in PTs [17] but epithelial metaplasia is uncommon. Apocrine and squamous changes can occur but malignant epithelial transformation, as reported in the present case, is exceptionally rare. This case adds to the literature of 38 cases in 31 reports $[1,4-$ 33] of carcinoma arising either within and/or in association with PT (Table 1). It demonstrates the range of changes 


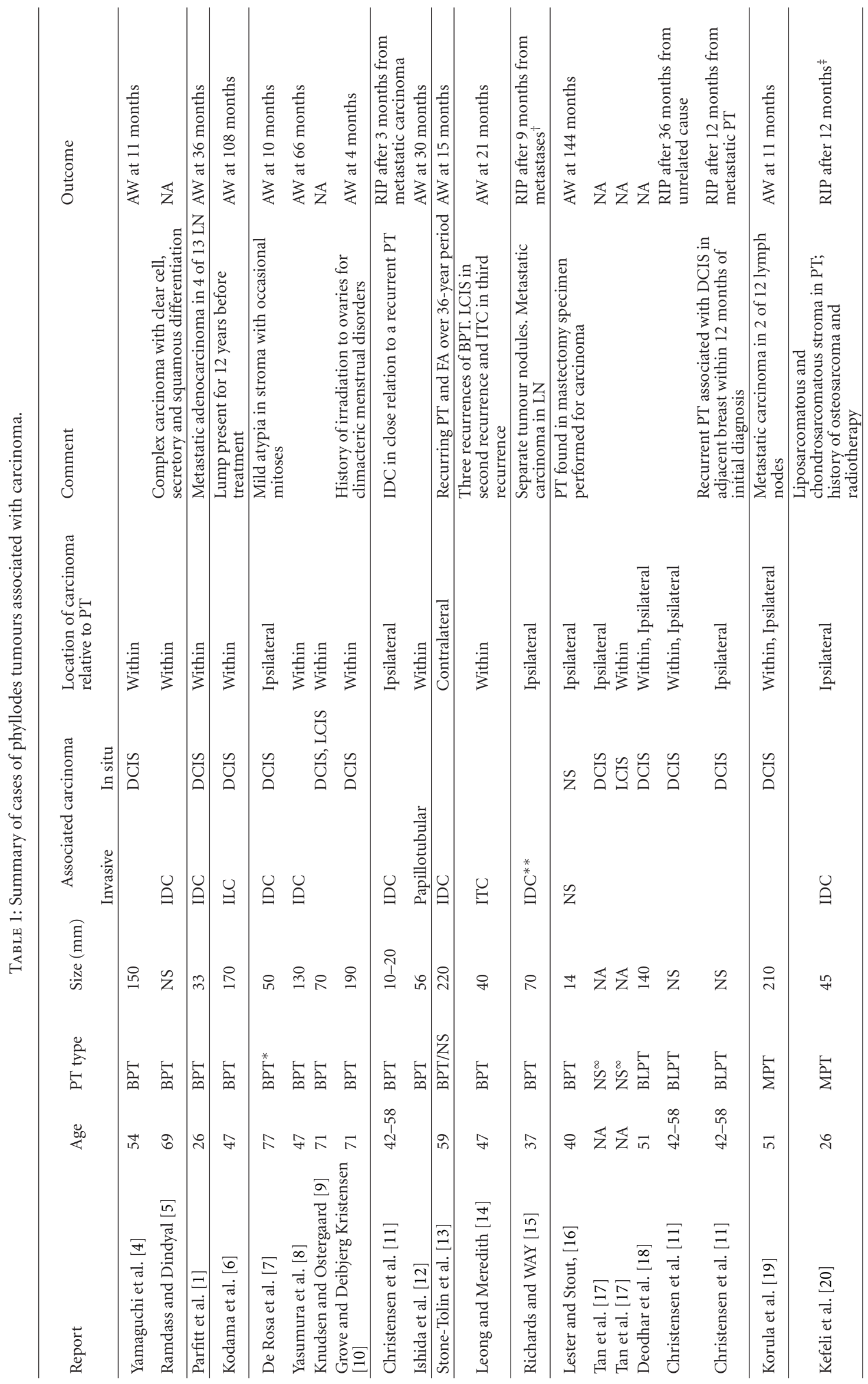




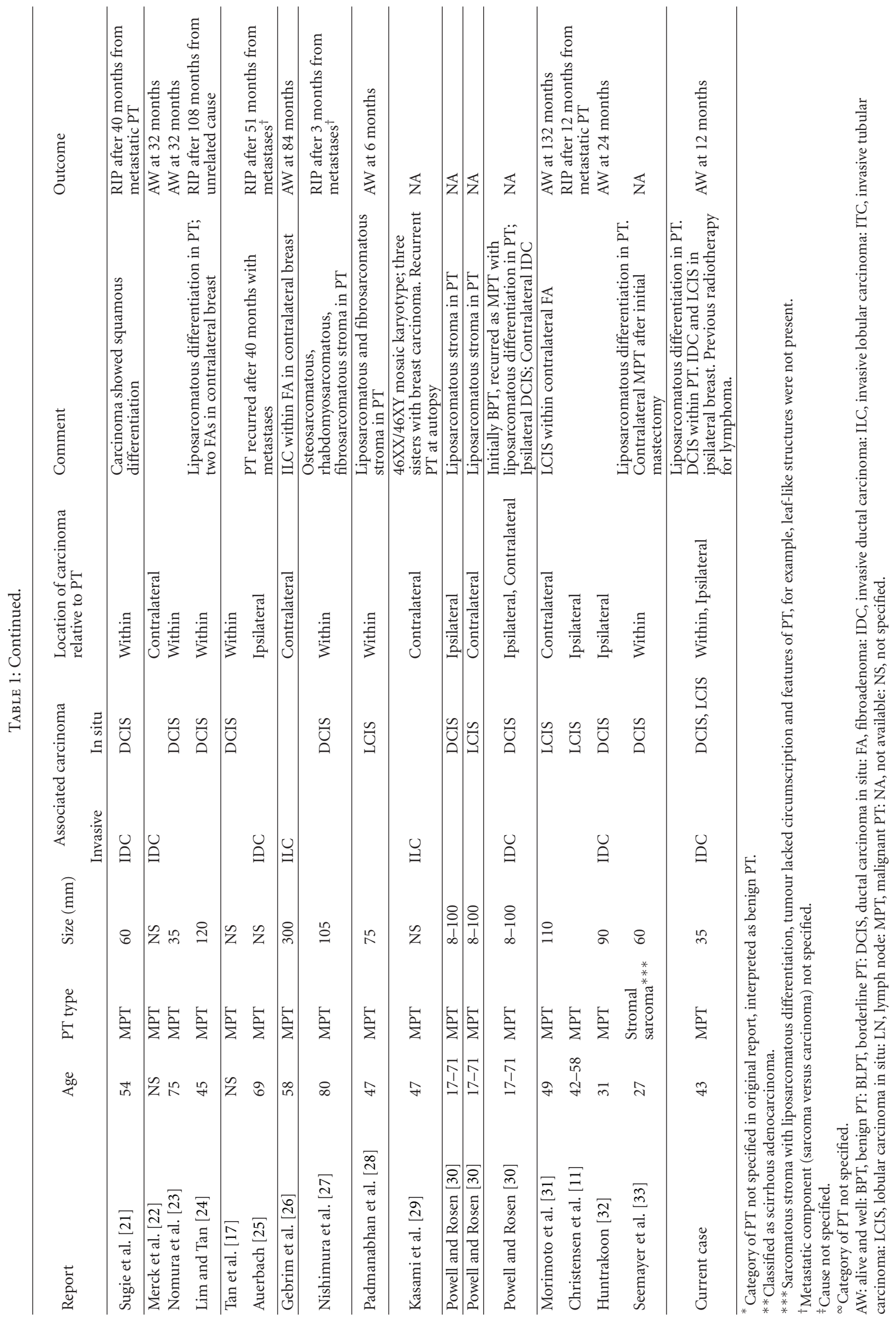




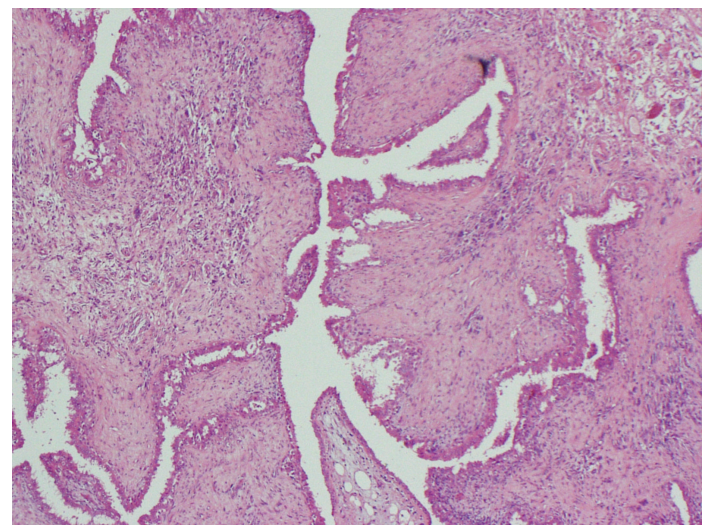

(a)

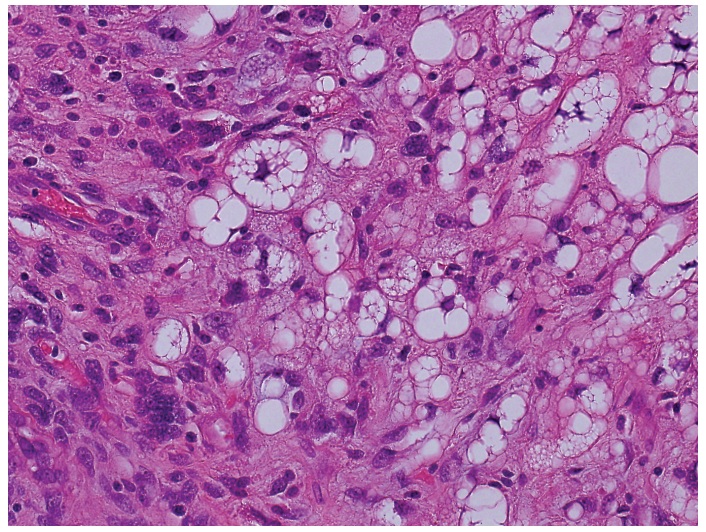

(c)



(b)

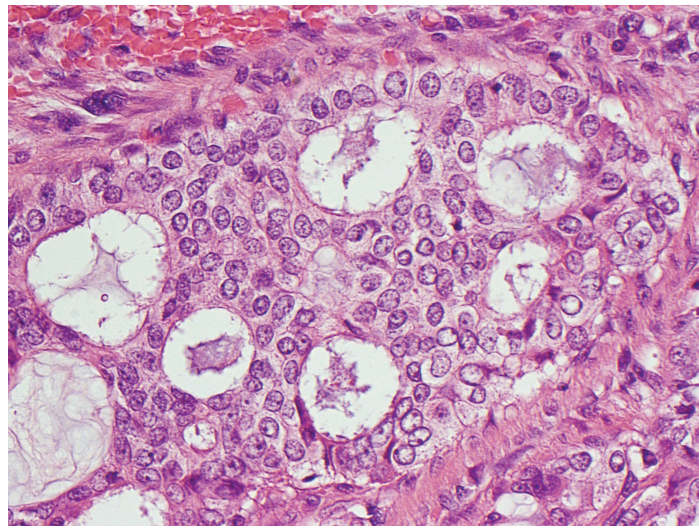

(d)

FIgURe 2: Pathological features within phyllodes tumour: (a) leaf-like architecture of the phyllodes tumour (H\&E, original magnification 40x); (b) fibrosarcomatous stroma (H\&E, original magnification 100x); (c) liposarcomatous differentiation in the stroma. Note the presence of lipoblasts (H\&E, original magnification 200x); (d) DCIS, intermediate nuclear grade (H\&E, original magnification 400x).

that can be seen in association with a PT, including both liposarcomatous stroma and DCIS within the tumour and DCIS, LCIS, and invasive carcinoma in the peritumoural ipsilateral breast.

Malignant epithelial elements are reported in all categories of PT. In situ and invasive carcinoma may involve the tumour itself and/or coexist with a PT elsewhere in the same or contralateral breast. Carcinoma is most commonly reported $(n=19)$ in malignant PTs. In the malignant PT category, carcinoma occurred more frequently in the ipsilateral $[11,20,25,30,32]$ or contralateral $[22,26,29-31]$ breast rather than within the confines of the PT $(n=7)[17$, $21,23,24,27,28,33$. Coexisting carcinoma in malignant PTs is more commonly ductal (invasive ductal carcinoma, $n$ $=6$; DCIS, $n=10$ ) than lobular phenotype (invasive lobular carcinoma, $n=2$; LCIS, $n=4)$ [11, 17, 19-33]. Heterologous stroma, most often liposarcomatous differentiation, was common in malignant PTs where carcinoma was also found $(n=8,47 \%)[20,21,24,27,30,33]$. From the available data, five patients with malignant PTs with coexisting carcinoma died from metastatic PT, between three months to 51 months after diagnosis [11, 20,21, 25, 27], and one patient had metastatic carcinoma in two lymph nodes [19].
Carcinoma is less common in benign PTs compared with malignant PTs, given the higher prevalence of the former. Coexistent carcinoma is reported in 16 benign PTs (including four of unspecified malignant potential) $[7,13,17]$ and in three of these cases it was only seen in a recurrence of a benign PT $[11,13,14]$. Carcinoma was found more commonly within the benign PT $(n=10)[1,4-6,8-10,12$, $14,17]$ rather than peripheral to it in the ipsilateral $(n=5)[7$, $11,15-17$ ] or contralateral breast $(n=1)$ [13]. The majority of these carcinomas $(n=10)$ were invasive, with or without an in situ component and most were ductal NST type ( $n=$ 6) $[1,7,8,11,13,15]$ with individual reports of invasive lobular [6], papillotubular [12], and tubular carcinoma [14]. One case had both invasive squamous carcinoma and invasive ductal carcinoma showing clear cell, secretory, and squamous differentiation [5]. Two patients died from breast disease $[11,15]$, one of which had metastatic carcinoma in lymph nodes [15]. Another patient had metastatic carcinoma in four lymph nodes but remained alive and well at follow-up [1].

There are only three reports of carcinoma (DCIS) arising in association with borderline PT. Two patients had DCIS both within the PT and in the ipsilateral breast $[11,18]$, one 


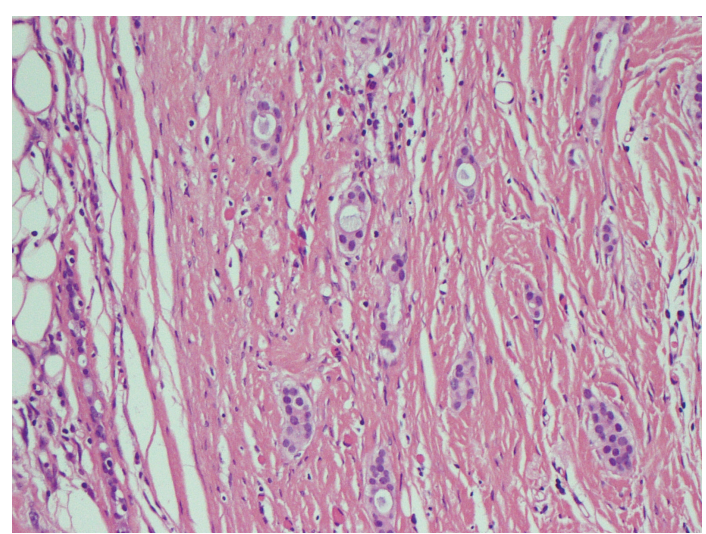

(a)

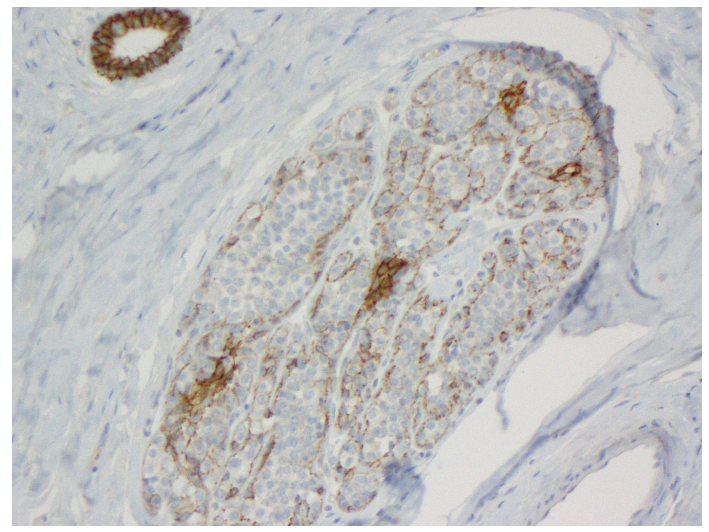

(c)

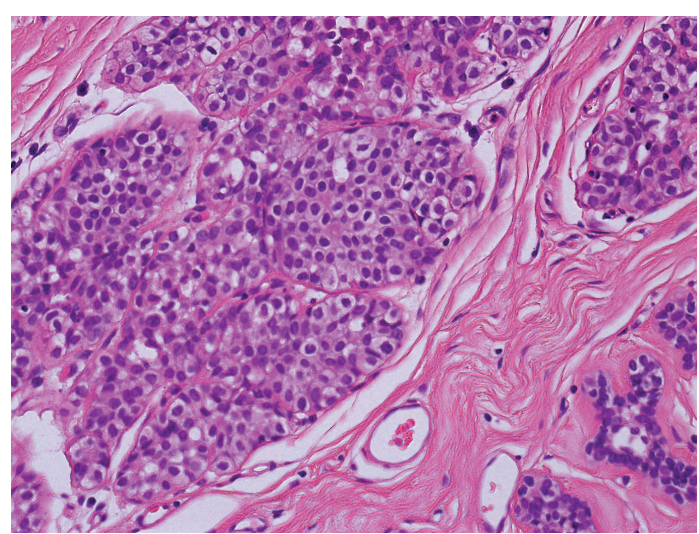

(b)

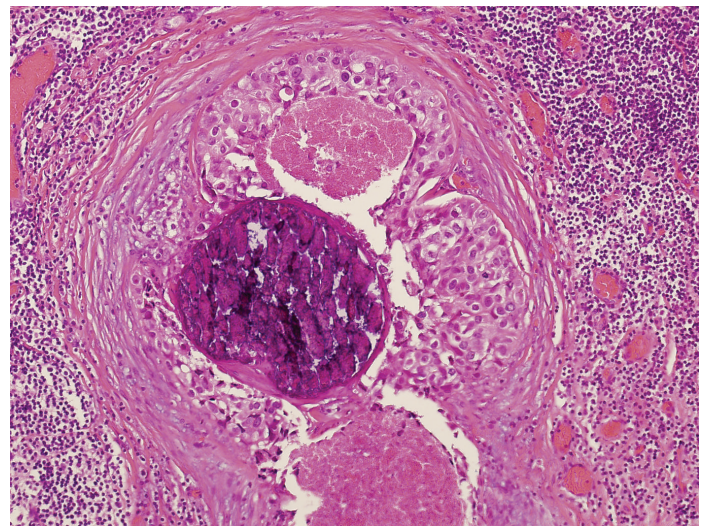

(d)

FIgURE 3: Pathological features in ipsilateral breast peripheral to phyllodes tumour(a)Invasive tubular carcinoma (H\&E, original magnification 200x); (b) LCIS (H\&E, original magnification 200x); (c) LCIS showing downregulation in E-Cadherin staining (E-Cadherin, original magnification 200x); (d) DCIS, high nuclear grade with comedonecrosis and calcification (H\&E, original magnification 200x).

of whom died within three years from unrelated causes. In the other case, DCIS occurred in the ipsilateral breast at the time of diagnosis of a recurrent PT and death occurred from metastatic PT one year after the recurrence [18].

The molecular events involved in transformation and progression of PTs are largely unknown, and the rarity of coexisting epithelial malignancy makes it difficult to draw conclusions about the aetiological association between the carcinoma and PT in these cases. Genetic aberrations have been consistently demonstrated in PTs with increasing frequency from benign to borderline to malignant PTs. Most studies to date focused on stromal alterations and showed recurrent copy number gains and losses at $+1 \mathrm{q},-13 \mathrm{q},-6 \mathrm{q}$, +5 , and $-10 \mathrm{p}$ [37-39]. Where epithelium was evaluated separately, distinct molecular alterations were demonstrated in the Wnt2-APC-B-catenin pathway, and a role was postulated for both stroma and epithelium in the neoplastic process [40, 41]. It is unclear, however, if the malignant transformation of epithelium results from stromal-epithelial interactions within the PT or if it represents cancerisation of a PT by carcinoma arising in the duct system peripheral to the PT. The latter may play a role in cases where carcinoma is present within the PT as well as peripheral to it in the ipsilateral breast. The finding of carcinoma in benign, borderline, and malignant PT and within fibroadenomas suggests that it is unlikely to be directly related to the number of genetic aberrations in the different categories of PT. A role of exogenous carcinogen exposure in malignant transformation merits consideration as an aetiological agent because two patients $[10,20]$ in addition to the present case had a history of previous radiation. In all cases the radiotherapy field was remote from the breast which suggest that it is likely to be incidental.

The present case taken together with those in the literature demonstrates the diverse pathology that can be found with PTs, both within the tumour and in the surrounding and/or contralateral breast. The distinction between a malignant PT with coexisting carcinoma and a metaplastic carcinoma or carcinosarcoma should be considered in the diagnosis as these entities are managed differently and the distinction affects patient outcome. Carcinosarcomas have mixed malignant epithelial and stromal components with the latter showing no reactivity for epithelial immunohistochemical markers. While the present case fulfils these criteria, the characteristic leaf-like structure with malignant heterologous differentiation favours a diagnosis of malignant PT 
with coexisting carcinoma rather than a true carcinosarcoma. Carcinomas coexisting with PTs are generally diagnosed incidentally on the wide local excision, and the prognosis is dictated by the category of PT. In the present case, the malignant PT and not the carcinomatous elements will have the greatest impact on prognosis. However, the presence of coexistent carcinoma must be taken into account in management decisions, especially for benign and borderline PTs. Wider adequacy of excision of carcinoma, as in this case, and lymph node sampling should be considered.

\section{References}

[1] J. R. Parfitt, C. Armstrong, F. O’Malley, J. Ross, and A. B. Tuck, "In-situ and invasive carcinoma within a phyllodes tumor associated with lymph node metastases," World Journal of Surgical Oncology, vol. 2, article 46, 2004.

[2] F. Tavassoli and P. Devilee, "Fibroepithelial tumours," in World Health Organization Classification of Tumours of the Breast and Female Genital Organs, pp. 99-103, IARC Press, Lyon, France, 2003.

[3] D. L. Page, I. O. Ellis, and C. W. Elston, "Histologic grading of breast cancer. Let's do it," American Journal of Clinical Pathology, vol. 103, no. 2, pp. 123-124, 1995.

[4] R. Yamaguchi, M. Tanaka, Y. Kishimoto, K. Ohkuma, M. Ishida, and M. Kojiro, "Ductal carcinoma in situ arising in a benign phyllodes tumor: report of a case," Surgery Today, vol. 38, no. 1, pp. 42-45, 2008.

[5] M. J. Ramdass and S. Dindyal, "Phyllodes breast tumour showing invasive squamous-cell carcinoma with invasive ductal, clear-cell, secretory, and squamous components," Lancet Oncology, vol. 7, no. 10, p. 880, 2006.

[6] T. Kodama, K. Kameyama, M. Mukai, H. Sugiura, T. Ikeda, and Y. Okada, "Invasive lobular carcinoma arising in phyllodes tumor of the breast," Virchows Archiv, vol. 442, no. 6, pp. 614 616, 2003.

[7] G. de Rosa, G. Ferrara, P. Goglia, C. Chicas, and P. Zeppa, "In situ and microinvasive carcinoma with squamoid differentiation arising in a phyllodes tumor: report of a case," Tumori, vol. 75, no. 5, pp. 514-517, 1989.

[8] T. Yasumura, S. Matsui, T. Hamajima, et al., "Infiltrating ductal carcinoma developing within cystosarcoma phyllodes-a case report," Japanese Journal of Surgery, vol. 18, no. 3, pp. 326329, 1988.

[9] P. J. T. Knudsen and J. Ostergaard, "Cystosarcoma phylloides with lobular and ductal carcinoma in situ," Archives of Pathology and Laboratory Medicine, vol. 111, no. 9, pp. 873875, 1987.

[10] A. Grove and L. Deibjerg Kristensen, "Intraductal carcinoma within a phyllodes tumor of the breast: a case report," Tumori, vol. 72, no. 2, pp. 187-190, 1986.

[11] L. Christensen, M. Nielsen, and P. M. Madsen, "Cystosarcoma phyllodes: a review of 19 cases with emphasis on the occurrence of associated breast carcinoma," Acta Pathologica Microbiologica et Immunologica Scandinavica, Section A, vol. 94, no. 1, pp. 35-41, 1986.

[12] T. Ishida, M. Izuo, and T. Kawai, "Breast carcinoma arising in cystosarcoma phyllodes: report of a case with a review of the literature," Japanese Journal of Clinical Oncology, vol. 14, no. 1, pp. 99-106, 1984.

[13] K. Stone-Tolin, E. W. Pollak, W. Dorzab, and J. Printz, "Recurring cystosarcoma phyllodes associated with breast carcinoma," Southern Medical Journal, vol. 75, no. 7, pp. 881$884,1982$.

[14] A. S. Y. Leong and D. J. Meredith, "Tubular carcinoma developing within a recurring cystosarcoma phyllodes of the breast," Cancer, vol. 46, no. 8, pp. 1863-1867, 1980.

[15] W. G. Richards and R. W. WAY, "Co-existent cystosarcoma phyllodes and schirrhous adenocarcinoma of the breast," Wisconsin Medical Journal, vol. 62, pp. 425-428, 1963.

[16] J. Lester and A. P. Stout, "Cystosarcoma phyllodes," Cancer, vol. 7, no. 2, pp. 335-353, 1954.

[17] P.-H. Tan, T. Jayabaskar, K.-L. Chuah, et al., "Phyllodes tumors of the breast: the role of pathologic parameters," American Journal of Clinical Pathology, vol. 123, no. 4, pp. 529-540, 2005.

[18] K. K. Deodhar, J. B. Baraniya, K. N. Naresh, S. R. Shinde, and R. F. Chinoy, "Cancerization of phyllodes tumour," Histopathology, vol. 30, no. 1, pp. 98-99, 1997.

[19] A. Korula, J. Varghese, M. Thomas, F. Vyas, and A. Korula, "Malignant phyllodes tumour with intraductal and invasive carcinoma and lymph node metastasis," Singapore Medical Journal, vol. 49, no. 11, pp. e318-e321, 2008.

[20] M. Kefeli, L. Yildiz, I. Akpolat, P. Balci, and N. Ozen, "The coexistence of invasive ductal carcinoma and malignant phyllodes tumor with liposarcomatous and chondrosarcomatous differentiation in the same breast in a post-osteosarcoma case," Pathology Research and Practice, vol. 204, no. 12, pp. 919-923, 2008.

[21] T. Sugie, E. Takeuchi, F. Kunishima, F. Yotsumoto, and Y. Kono, "A case of ductal carcinoma with squamous differentiation in malignant phyllodes tumor," Breast Cancer, vol. 14, no. 3, pp. 327-332, 2007.

[22] B. Merck, P. Cansado Martinez, M. Pérez Ramos, N. Martínez Banaclocha, F. J. Lacueva Gómez, and R. Calpena, "Infiltrating ductal carcinoma and synchronous malignant phyllodes tumour: diagnostic and therapeutic approaches," Clinical and Translational Oncology, vol. 8, no. 11, pp. 830-832, 2006.

[23] M. Nomura, Y. Inoue, S. Fujita, et al., "A case of noninvasive ductal carcinoma arising in malignant phyllodes tumor," Breast Cancer, vol. 13, no. 1, pp. 89-94, 2006.

[24] S. M. Lim and P. H. Tan, "Ductal carcinoma in situ within phyllodes tumour: a rare occurrence," Pathology, vol. 37, no. 5, pp. 393-396, 2005.

[25] L. Auerbach, "Concomitant phyllodes tumour and homolateral breast cancer," Lancet Oncology, vol. 3, no. 5, p. 279, 2002.

[26] L. H. Gebrim, J. R. Bernardes Júnior, A. C. Nazário, C. Kemp, and G. R. Lima, "Malignant phyllodes tumor in the right breast and invasive lobular carcinoma within fibroadenoma in the other: case report," Sao Paulo Medical Journal, vol. 118, no. 2, pp. 46-48, 2000.

[27] R. Nishimura, T. Hasebe, S. Imoto, and K. Mukai, "Malignant phyllodes tumour with a noninvasive ductal carcinoma component," Virchows Archiv, vol. 432, no. 1, pp. 89-93, 1998.

[28] V. Padmanabhan, J. E. Dahlstrom, G. C. Chong, and G. Bennett, "Phyllodes tumor with lobular carcinoma in situ and liposarcomatous stroma," Pathology, vol. 29, no. 2, pp. 224226, 1997.

[29] M. Kasami, M. Yoshida, J. Isogaki, et al., "Bilateral breast tumors, malignant phyllodes tumor and invasive lobular carcinoma in a 46,XX/46,XY mosaic female with family history of breast cancer," Pathology International, vol. 47, no. 2-3, pp. 147-154, 1997.

[30] C. M. Powell and P. P. Rosen, "Adipose differentiation in cystosarcoma phyllodes: a study of 14 cases," American Journal of Surgical Pathology, vol. 18, no. 7, pp. 720-727, 1994. 
[31] T. Morimoto, T. Tanaka, K. Komaki, et al., "The coexistence of lobular carcinoma in a fibroadenoma with a malignant phyllodes tumor in the opposite breast: report of a case," Surgery Today, vol. 23, no. 7, pp. 656-660, 1993.

[32] M. Huntrakoon, "Malignant cystosarcoma phyllodes with simultaneous carcinoma in the ipsilateral breast," Southern Medical Journal, vol. 77, no. 9, pp. 1176-1178, 1984.

[33] T. A. Seemayer, G. Tremblay, and H. Shibata, "The unique association of mammary stromal sarcoma with intraductal carcinoma," Cancer, vol. 36, no. 2, pp. 599-605, 1975.

[34] P. A. Isotalo, R. L. George, R. Walker, and S. K. SenGupta, "Malignant phyllodes tumor with liposarcomatous differentiation," Archives of Pathology and Laboratory Medicine, vol. 129, no. 3, pp. 421-422, 2005.

[35] R. M. Austin and W. B. Dupree, "Liposarcoma of the breast: a clinicopathologic study of 20 cases," Human Pathology, vol. 17, no. 9, pp. 906-913, 1986.

[36] P. P. Rosen, "Fibroepithelial neoplasms," in Rosen's Breast Pathology, pp. 176-200, Lippincott Williams \& Wilkins, Philadelphia, Pa, USA, 2nd edition, 2001.

[37] A. M. Jones, R. Mitter, R. Springall, et al., "A comprehensive genetic profile of phyllodes tumours of the breast detects important mutations, intra-tumoral genetic heterogeneity and new genetic changes on recurrence," Journal of Pathology, vol. 214, no. 5, pp. 533-544, 2008.

[38] A. Kuijper, A. M. Snijders, E. M. J. J. Berns, et al., "Genomic profiling by array comparative genomic hybridization reveals novel DNA copy number changes in breast phyllodes tumours," Cellular Oncology, vol. 31, no. 1, pp. 31-39, 2009.

[39] M. Laé, A. Vincent-Salomon, A. Savignoni, et al., "Phyllodes tumors of the breast segregate in two groups according to genetic criteria," Modern Pathology, vol. 20, no. 4, pp. 435-444, 2007.

[40] E. J. Sawyer, A. M. Hanby, P. Ellis, et al., "Molecular analysis of phyllodes tumors reveals distinct changes in the epithelial and stromal components," American Journal of Pathology, vol. 156, no. 3, pp. 1093-1098, 2000.

[41] E. J. Sawyer, A. M. Hanby, A. J. Rowan, et al., "The Wnt pathway, epithelial-stromal interactions, and malignant progression in phyllodes tumours," Journal of Pathology, vol. 196, no. 4, pp. 437-444, 2002. 


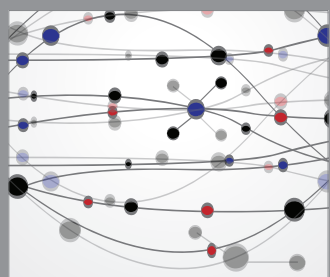

The Scientific World Journal
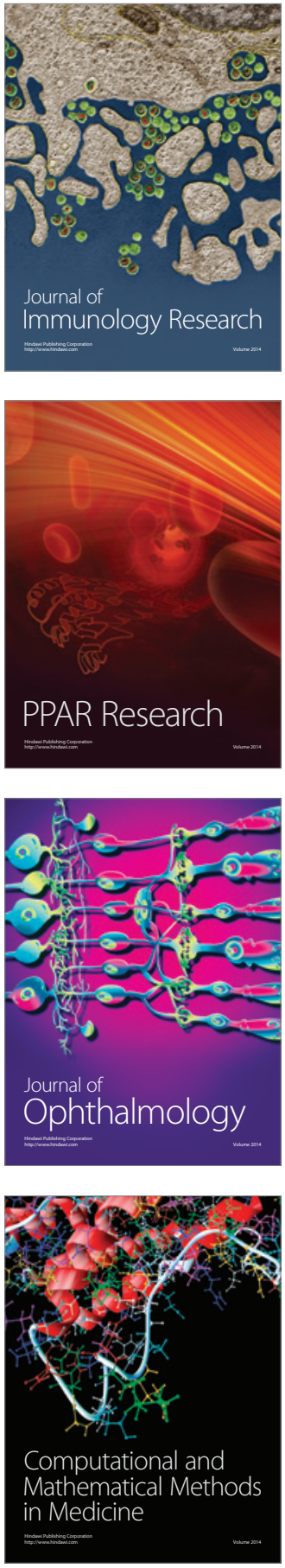

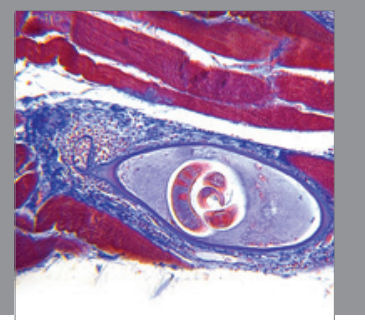

Gastroenterology

Research and Practice
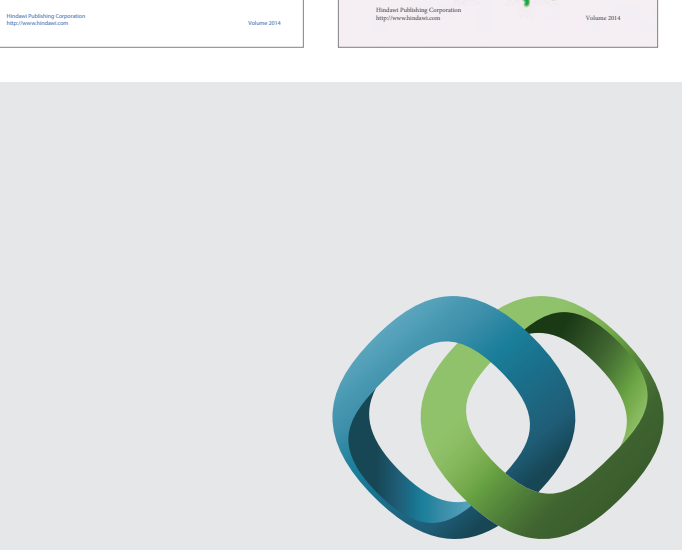

\section{Hindawi}

Submit your manuscripts at

http://www.hindawi.com
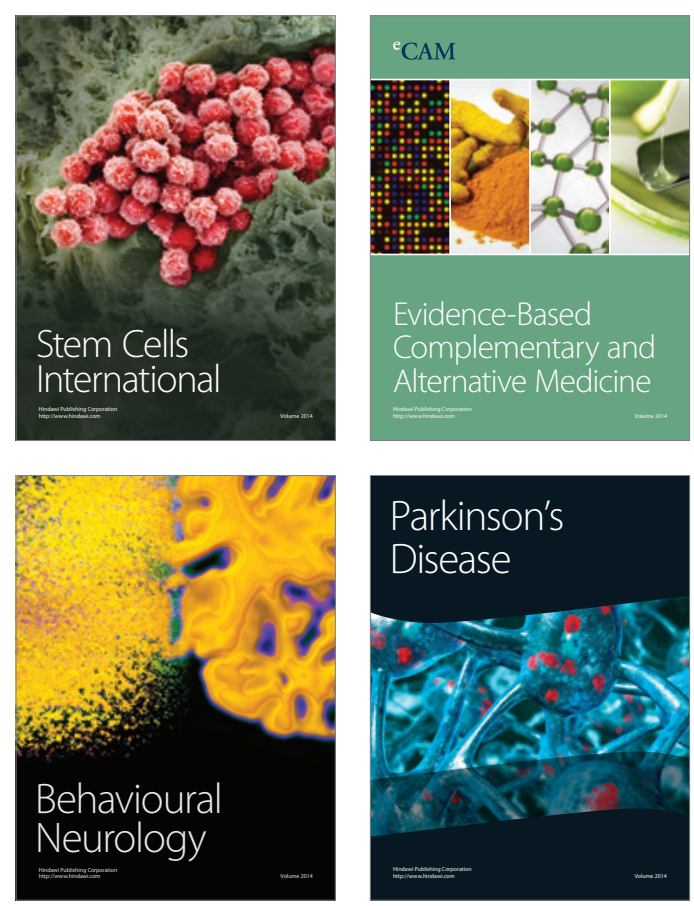

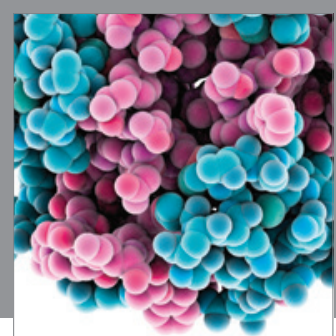

Journal of
Diabetes Research

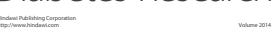

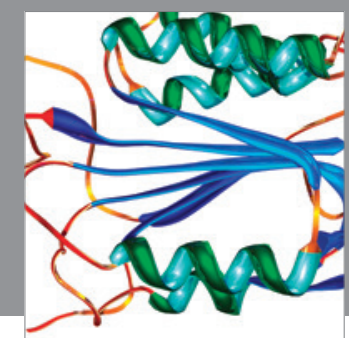

Disease Markers
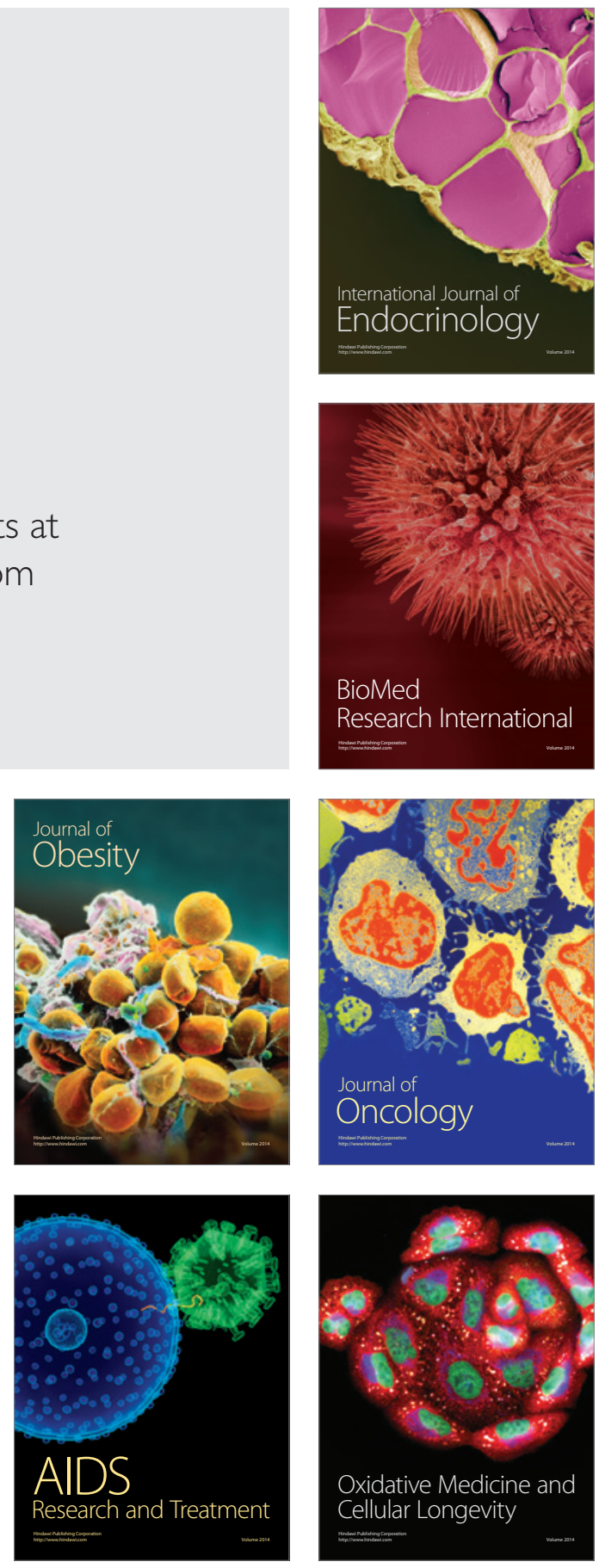